\title{
Pluralismus und Antipluralismus
}

\section{Dorothée de Nève}

Justus-Liebig-Universität Gießen, Institut für Politikwissenschaft, Deutschland

E-Mail: dorothee.de-neve@sowi.uni-giessen.de

\section{Zusammenfassung}

Das Paradox der aktuellen Forschung besteht darin, dass Pluralismus einerseits ein Kernbegriff der Politikwissenschaft als Demokratiewissenschaft darstellt, andererseits eine fundierte Auseinandersetzung damit in der aktuellen Forschung kaum stattfindet. Der nachfolgende Beitrag erläutert die relevanten Perspektiven der bisherigen Forschung in verdichteter Form und spricht sich angesichts des aktuellen Erkenntnisstandes dafür aus, die Bandbreite antipluralistischer Tendenzen nicht auf die Analyse des Extremismus zu begrenzen.

\section{Schlüsselwörter}

Pluralismus, Antipluralismus

\section{Pluralism and antipluralism}

\section{Abstract}

The paradox of the current research is that pluralism is a core concept of political science as science on democracy. At the same time, this key concept in current research is still vague. The following article explains the relevant perspectives of previous research in a condensed form and favors, in view of the current state of knowledge, that the analysis of the bandwidth of antipluralistic tendencies should not be limited to the study of extremism.

\section{Keywords}

Pluralism, anti-pluralism

\section{Danksagung}

Ich bedanke mich bei Verena Schäfer-Nerlich, Jan Weyland und Tina Musil für wertvolle Hinweise und Anregungen für die Arbeit an diesem Beitrag.

The author has declared that no competing interests exist. 
Die drei nachfolgenden Beiträge zum Thema Antipluralismus entstanden in der Zusammenarbeit der Autorinnen im Kontext der Dreiländertagung „Politik der Vielfalt" in Innsbruck (20I3). In einem gemeinsamen Panel hinterfragten die Autorinnen die normativen Prämissen der Pluralismusund Antipluralismusforschung und diskutierten neue empirische Befunde politikwissenschaftlicher Forschung.

Betrachtet man die aktuelle politikwissenschaftliche Debatte um den Pluralismusbegriff, so erscheint die Situation an sich paradox: Pluralismus wird als Kernbegriff der Politikwissenschaft identifiziert, zumindest wenn man diese Disziplin in der Tradition der Demokratiewissenschaft verortet (Zeuner 1989, I28). In der so genannten freiheitlichdemokratischen Grundordnung manifestiert sich Pluralismus als der normative Bezugspunkt für Freiheit und Vielfalt individueller Meinungen, Interessen und Lebensentwürfe. Wenn man sich Ernst Fraenkels Semantik bedient, so geht es um nichts Geringeres als um die Lebensfähigkeit der Demokratie (Fraenkel I958', 30). Der Begriff der pluralistischen Demokratie verdeutlicht die prinzipiell positive Konnotation von Pluralismus als Merkmal der Demokratie.

Dies bildet sich freilich zumindest in der aktuellen theoretischen Auseinandersetzung mit dem Begriff nur partiell ab. In Beiträgen zu dem Pluralismusbegriff finden sich nur selten klare Definitionen. Stattdessen werden Pluralismuskonzepte - etwa als Gegensatz zum monistischen Staat - konzipiert und Erscheinungsformen des Pluralismus beschrieben (Hättich 1980, I09; Rudzio 2015, 65). Pluralismusforschung ist insofern über weite Strecken deskriptiv, präskriptiv und empirisch. Obwohl derzeit eine gewisse akademische Faszination für Grundbegriffe der Demokratie wie Gerechtigkeit oder Solidarität neu entdeckt wird (Brockmann 2015; Krück/Merkel 2004, 85), so scheint sich dieses Interesse bislang jedenfalls (noch?) nicht auf den Pluralismusbegriff zu erstrecken.

Bei dem Versuch, angesichts dieser ungünstigen Ausgangslage dennoch den Begriffskern von Pluralismus zu identifizieren, bietet der etymologische Ursprung erste Ansatzpunkte. Das lateinische Wort pluralis steht für mehrfach bzw. vielfach, der Begriff des Pluralismus insofern für Vielfalt. Die Pluralismustheorie unterscheidet zwischen zwei Varianten der Deutung: Erstens die Lesart des Pluralismus als Gruppenpluralismus, der den Alleinzuständigkeitsanspruch des Herrschaftssystems herausfordert und als Instrument politischer und gesellschaftlicher Innovation gilt, indem vielgliedrige, nicht monistische politische Ordnungsstrukturen etabliert werden (Steffani I980, 33). Mit dieser Dimension des Pluralismusbegriffs beschäftigt sich mitunter die Forschung zur Institutionalisierung kollektiver Interessen, zur Gewaltenteilung und zu den Strategien der Interdepenzbewältigung in der modernen Governanceforschung. Zweitens geht es um den Pluralismus als Produkt des Individualismus und sozialer Differenzierung. Dabei handelt es sich um ein philosophisches Weltbild, das von der Existenz gesellschaftlicher Heterogenität ausgeht.
Soziale Heterogenität - und dies ist insbesondere für die sozialwissenschaftliche Forschung von Interesse - entsteht durch institutionelle, sozioökonomische, kulturelle und soziale (Lebens-)Kontexte. Indem verschiedene Faktoren letztlich gesellschaftspolitische Heterogenität generieren, entsteht freilich nicht ein allumfassendes Ganzes, denn es gibt keine deterministische Disposition, die beispielsweise aus einem spezifischen sozioökonomischen Setting bestimmter Faktoren einen beschreibbaren Typus hervorbringt. Vielmehr stehen differierende Faktoren in sich verändernden Beziehungen zueinander. Aus diesem spezifischen Verständnis von Pluralismus folgt, dass pluralistische gesellschaftliche Wirklichkeit veränderbar und gestaltbar ist. Allgemein gehen gesellschaftswissenschaftliche Theorien zwar oft davon aus, dass etwa durch Prozesse der Modernisierung, Säkularisierung oder Globalisierung Pluralismus in unserer Gesellschaft mehr geworden sei, dies mag freilich auch einer gewissen Geschichtsvergessenheit geschuldet sein (vgl. Müller 2006, 406).

Die politikwissenschaftliche Pluralismusforschung weist eine normative Komponente auf. Heterogenität bzw. Pluralismus gilt nicht nur als wünschenswert und legitim, sondern notwendig, denn Wettbewerb gilt als der Demokratie inhärent (Musil 2005, 385). Dies wird insbesondere im Kontext der Demokratietheorie deutlich: „Wie sich der totale Staat aus der Negation des Pluralismus rechtfertigt, so rechtfertigt sich die freiheitliche Demokratie vornehmlich aus dem Bekenntnis zum Pluralismus" (Steffani 1980, 37).

Mit der Existenz des Pluralismus sind freilich Konflikte unvermeidlich. Zur Konfliktlösung bedarf es einer prozessualen Einigkeit über Prinzipien, Normen und Verfahren (Schubert I998, 483). Jenseits der unvermeidlichen und als legitim erachteten Konflikte wird Pluralismus indes auch als eine Ursache für Krisen wahrgenommen. Der Mensch brauche, so die Annahme, angesichts pluraler Lebenswelten Orientierung. Pluralistische Lebenswelten führen angeblich zu Orientierungslosigkeit und Sinnkrisen, wenn Beliebigkeit und verschiedene, konkurrierende Morale die "gesunde" geistige Orientierung gefährden. Die Suche nach den gemeinsamen Elementen werde so erschwert - wenn nicht gar verhindert (Berger 1995, 74). Dieser Logik folgend wird die Notwendigkeit einer "geistigen Orientierung" in einer pluralen Lebenswelt betont (Weidenfeld (Hg.) I994, 5). Bornemann spricht von Neurosen, die in pluralistischen Gesellschaften erzeugt würden (Bornemann 1997, 52). Auch wenn das Bemühen solcher Krankheitsbilder an sich problematisch ist, so wird damit angedeutet, dass es sich um kontextbedingte Reaktionen handelt, die (vermeintlich) Störungen im System hervorrufen. Pluralismus selbst wird als Bedrohung wahrgenom- 
men, indem der Konflikt als Gefahr stilisiert wird. Pluralismus führe zur sozialen Desintegration und damit logisch zu einer Anhäufung sozialer Konflikte (Berger 1995, 74).

Befürchtungen dieser Art basieren auf der Annahme, dass Gesellschaften einer gemeinsamen Wertebasis bedürften, die durch Pluralismus latent in Frage gestellt werde (Durkheim 1899). Sie kulminieren letztlich in den Vorstellungen, dass Pluralismus die Gesellschaft zerstöre (Oberreuter 1980, I23), bis hin zu dem Szenario des "clash of civilizations“(Huntington 1993), das die unvermeidliche Eskalation des Konflikts prognostiziert. Die als bedrohlich wahrgenommenen Konflikte, die als Folge des Pluralismus erachtet werden, bilden die Grundlage für antipluralistische Argumentationslogiken. Antipluralismus ist in diesem Sinne die vermeintlich zielführende Strategie der Konfliktvermeidung und Garant für Stabilität und Ordnung.

Insofern wird Pluralismus in demokratischen Gesellschaften offenbar also nicht nur als legitim und notwendig, sondern zuweilen auch als latent problematisch oder gar bedrohlich wahrgenommen. Dieses Spannungsverhältnis manifestiert sich außerdem in der Erwartung hinsichtlich dynamischer Entwicklungen und Veränderungen einerseits und jener nach Ordnung, Berechenbarkeit und Stabilität andererseits. Damit verbunden ist auch die in unseren Gesellschaften und Kulturen offensichtlich tief verankerte Sehnsucht nach Harmonie, die als Widerspruch zu dem pluralistischen Weltbild konzipiert wird. Der Pluralismus, die Bewegung und Veränderung wird wohl allzu oft nicht als harmonisch, sondern als irritierend und störend wahrgenommen. Die Einheit bzw. Einheitlichkeit wird zu einem Ideal stilisiert - was sich etwa in Deutschland mitunter in der fragwürdigen Debatte um die so genannte "Leitkultur" zeigt - und offenbart sich darüber hinaus in der vermeintlich unverrückbaren Forderung nach Gemeinwohlorientierung. Pluralistische Demokratie ist in Fraenkels Verständnis nur lebensfähig, wenn sie eine Organisation des Gesamtvolkes darstellt. Diese Organisation steht dann sozusagen über den Partikulargruppen und deren pluralistischem Charakter (Fraenkel 20II, 338). Die Forderung nach Gemeinwohlorientierung nimmt zuweilen geradezu abstruse Formen an, insbesondere wenn sozialen, ethnischen oder religiösen Akteuren, die Partikularinteressen vertreten - was angesichts des demokratischen Wettbewerbs funktionslogisch nicht nur plausibel, sondern auch legitim erscheint - Gemeinwohlorientierung als Akt der Unterwerfung abverlangt wird. Dabei bleibt freilich oft unklar, wie sich diese Gemeinwohlorientierung letztlich begründet.

Ein weiteres Dilemma ist offenbar der Widerspruch zwischen Disposition und Wahlfreiheit. Wenn gesellschaftliche Heterogenität entsteht, dann steht latent die Frage im Raum, ob dieser Prozess vorbestimmt ist oder Entscheidungsfreiheit besteht. Insbesondere in der empirischen Politikwissenschaft dominiert wohl meist die Vorstellung spezifischer Dispositionen. Dieses Deutungsmuster findet sich unter anderem in sozialstrukturellen und sozialpsychologischen Ansätzen der Wahlforschung wieder. Wenn man allerdings annimmt, dass Heterogenität in sich verändernden Lebenswelten entsteht und die individuelle und gesellschaftliche Wirklichkeit gestaltbar ist, werden politische Präferenzen der BürgerInnen und Phänomene wie Wahlverhalten noch viel schwerer erklärbar, als sie es ohnehin bereits sind.

Ein Spannungsverhältnis besteht auch hinsichtlich der Möglichkeiten, Heterogenität zu artikulieren beziehungsweise zu praktizieren. Ein praktisches Beispiel für diesen Konflikt sind die aktuellen Auseinandersetzungen um die Präsenz von Religion in der öffentlichen Sphäre und die Grenzen der Religionsfreiheit (vgl. de Nève 20II). „Aus Religionsfreiheit darf keine Religionsgleichheit werden" (Stern Online) - das war einer der kernigen Sätze, mit denen CDU-Politiker auf die Rede des deutschen Bundespräsidenten Wulff zum Islam in Deutschland reagierten. Die Idee von „simple equality“ wird verworfen. Stattdessen wird die Forderung artikuliert, Gleiches ungleich zu behandeln. Dies gilt jenseits der Auseinandersetzungen um Religion beispielsweise auch für Fragen der ethnischen, sexuellen oder ökonomischen Selbstbestimmung.

Tina Olteanu erläutert in ihrem Beitrag zu Antiziganismus und Homophobie, wie die diskursive Konstruktion von Identität wesentlich über die antipluralistische Abgrenzung funktioniert und diese Tendenzen Teil des Mainstreams geworden sind. Dabei zeigt Olteanu auf, wie der einst positiv konnotierte Begriff des Multikulturalismus inzwischen populistisch umgedeutet wird und nunmehr zuweilen als eine Gefährdung von Demokratie und Gesellschaft wahrgenommen wird. In ihrem Beitrag erschließt Olteanu neue Quellen, indem sie Postings auf Webseiten österreichischer Tageszeitungen als Instrumente politischer Partizipation analysiert.

Edma Ajanovic, Stefanie Mayer und Birgit Sauer widmen sich dem Antipluralismus in rechtsextremen bzw. rechtspopulistischen Diskursen in Österreich. Die Autorinnen zeigen auf, wie die Imagination einer einheitlichen, antipluralen Gemeinschaft räumlich konstruiert wird. Die Strategie der Ausgrenzung wird dabei über Nationalität, Kultur, Religion und Geschlecht sowie durch die Verknüpfung dieser Differenzkriterien konstruiert.

In ihrem Beitrag befasst sich Karin Liebhart mit visuellen Repräsentationen antipluralistischer Politik am Beispiel der Islam- und Muslimenfeindlichkeit in Österreich und der Schweiz. Dabei spielt der Begriff des Ethnopluralismus eine zentrale Rolle, der einerseits die Existenz kultureller Pluralität anerkennt, andererseits jedoch eine exklusivistische Position einnimmt. Liebhart zeigt, wie der Islam und die Muslime als krisen- und 
konfliktauslösend visuell repräsentiert werden und als Ursache für einen drohenden gesellschaftlichen Identitäts- und Orientierungsverlust stilisiert werden.

Die hier vorliegenden Beiträge machen deutlich, dass die Bandbreite antipluralistischer Tendenzen groß ist - sie reicht von Negieren über Ignorieren und Unterdrücken von Pluralismus bis hin zu Ausgrenzen und Zerstören. Antipluralistische Tendenzen werden wahlweise geschlechtlich, ethnisch, religiös, territorial, sozioökomisch, gesellschaftspolitisch o.ä. begründet. Dabei spielen Überlagerungen neuer und alter Stereotype und Feindbildkonstruktionen für Phänomene wie etwa Antisemitismus, Islamophobie, Homophobie, Xenophobie, Antiziganismus und Sexismus eine zentrale Rolle. Für die künftige Forschung scheint es ausgesprochen wichtig, dieses antipluralistische Weltbild nicht nur als Phänomen zu betrachten, das sich an den extremistischen Rändern unserer Gesellschaften verortet. Vielmehr handelt es sich um ein Weltbild, das sich in allen gesellschaftlichen Sphären - vom Staatsapparat bis zur Zivilgesellschaft und zu Unternehmen - wiederfindet. Insofern gilt es für die Politikwissenschaft auch künftig, die Erforschung des Antipluralismus nicht auf den Extremismus zu begrenzen.

Darüber hinaus werden die Potenziale künftiger empirischer politikwissenschaftlicher Forschung zum Thema Pluralismus und Antipluralismus im Anschluss an die hier vorliegenden Forschungsergebnisse darin gesehen, sich verstärkt mit den gesellschaftspolitischen Diskursen bzgl. der vermeintlich notwendigen Begrenzung des Pluralismus kritisch auseinanderzusetzen. Gegenwärtig werden demokratische Grundprinzipien durch Warnungen vor einem ausufernden Pluralismus und der mutmaßlichen Notwendigkeit, Pluralismus einzugrenzen und zu "domestizieren“, in Frage gestellt. Diese Tendenzen durchdringen unser politisches und gesellschaftliches System weitreichend und lassen eine neue systematische politikwissenschaftliche Analyse notwendig erscheinen.

\section{Literatur}

Bornemann, Ernst Wilhelm Julius (1997). Die Zukunft der Liebe, Frankfurt am Main.

Brockmann, Nils (2015). Zivilgesellschaftliche Akteure als Generatoren demokratieförderlicher Solidarität - Bloße Wunschvorstellung oder berechtigte Hoffnung?, in: Marianne Kneuer/Carlo Masala (Hg.): Solidarität. Politikwissenschaftliche Zugänge zu einem vielschichtigen Begriff. Sonderband der Zeitschrift für Politikwissenschaft, Baden-Baden, i.E.

de Nève, Dorothée (2011). Grenzen der Religionsfreiheit, in: Adrian Loretan (Hg.): Religionsfreiheit im Kontext der Grundrechte, Zürich, I63-I87.
Durkheim, Émile (I899). De la définition des phénomènes religieux, in: L’Année Sociologique, Vol. 2, I-28.

Fraenkel, Ernst (201I). Deutschland und die westlichen Demokratien, Baden-Baden.

Fraenkel, Ernst (1958). Die repräsentative und die plebiszitäre Komponente im demokratischen Verfassungsstaat, Tübingen.

Hättich, Manfred (1980). Pluralismus als Aufgabe, in: Heinrich Oberreuter (Hg.): Pluralismus, Opladen, I09-I25.

Krück, Mirko/Wolfgang Merkel (2004). Soziale Gerechtigkeit und Demokratie, in: Aurel Croissant/Gero Erdmann/Friedbert W. Rüb (Hg.): Wohlfahrtsstaatliche Politik in jungen Demokratien, Wiesbaden, 85-IIO.

Müller, Michael G. (2006). Der Consensus Sendomirensis - Geschichte eines Scheiterns?, in: Joachim Bahlcke/Karen Lamprecht/Hans-Christian Maner (Hg.): Konfessionelle Pluralität als Herausforderung. Koexistenz und Konflikt in Spätmittelalter und Früher Neuzeit. Festschrift für Winfried Eberhard zum 65. Geburtstag, Leipzig, 397-406.

Musil, Andreas (2005). Wettbewerb in der staatlichen Verwaltung, Tübingen.

Oberreuter, Heinrich (1980). Pluralismus: Grundlegung und Diskussion. Opladen.

Rudzio, Wolfgang (2015). Das politische System der Bundesrepublik Deutschland, Wiesbaden.

Schubert, Klaus (1998). Pluralismus, in: Dieter Nohlen, et al. (Hg.): Lexikon der Politik, Band 7 Politische Begriffe, München, 482-484.

Steffani, Winfried (1980). Pluralistische Demokratie Studien zur Theorie und Praxis, Opladen.

Stern Online (2010). Unions-Kritik am Bundespräsidenten. Religionsfreiheit darf keine Religionsgleichheit sein. 5. Oktober. Internet: http://www.stern.de/ politik/deutschland/unions-kritik-am-bundespraesidenten--religionsfreiheit-darf-keine-religionsgleichheit-sein--3528230.html (Zugriff: 22.6.2015).

Weidenfeld, Werner (Hg.) (1994). Orientierungsverlust - zur Bindungskrise der modernen Gesellschaft, Gütersloh.

Zeuner, Bodo (1989). Politikwissenschaft als Demokratiewissenschaft - ein vergessener Anspruch, in: Ulrich Albrecht (Hg.): Was heißt und zu welchem Ende betreiben wir Politikwissenschaft? Opladen, I28-I42.

\section{Autorin}

Dorothée de Nève ist Professorin für Politisches und Soziales System Deutschlands/Vergleich politischer Systeme am Institut für Politikwissenschaft an der JustusLiebig-Universität in Gießen. Ihre Forschungsschwerpunkte sind Partizipations- und Einstellungsforschung, Governance sowie Politik und Medien.. 